\title{
Application d'un système vidéo au suivi de la dynamique côtière d'un panache turbide de surface : Cas du panache de l'Adour
}

\author{
Damien DAILLOUX ${ }^{(1)}$, Denis MORICHON ${ }^{(2)}$, Stéphane ABADIE ${ }^{(3)}$ \\ (1) Doctorant, LaSAGeC ${ }^{2}$, Université de Pau et des Pays de l'Adour, \\ Anglet, FRANCE ; AZTI Tecnalia, Pasaia, Espagne \\ (2) Post Doctorant, LaSAGeC ${ }^{2}$, Université de Pau et des Pays de \\ l'Adour, Anglet, FRANCE \\ (3) Maître de Conférences, LaSAGeC ${ }^{2}$, Université de Pau et des Pays \\ de l'Adour, Anglet, FRANCE
}

\section{Résumé}

Les fleuves rejettent une quantité importante de matières en suspension (MES) dans les régions côtières. La présence de ces MES est souvent identifiable par une signature visuelle en forme de panache. L'exploitation de cette information est réalisée à partir d'un système de vidéo terrestre installé à Anglet. Ce dispositif permet d'obtenir automatiquement et à haute fréquence des données géoréférencées du contour du panache de l'Adour. La forme et la position du panache est comparée aux différents forçages (débit fluvial, marée et vent) susceptibles de contrôler sa dynamique. L'estuaire de l'Adour possède des caractéristiques pulsatiles induites par la marée. La force de Coriolis et le vent ont un impact majeur sur la direction de propagation du panache prés des côtes.

\begin{abstract}
$\underline{\text { Abstract }}$
Rivers reject a large quantity of suspended particule matter (SPM) in coastal areas. The presence of these SPM is often identify by a visual signature in the shape of a plume. The exploitation of this information is carried out from a terrestrial video system installed in Anglet. This device makes possible to obtain, automatically and at a high frequency, geo-referenced data of the Adour plume front. The shape and the position of the plume are compared with various forcings (river flow, tide and wind) likely to control its dynamics. The Adour estuary has pulse discharge characteristics inferred by tide. The Coriolis and wind forces have a major impact on the plume direction propagation close to the coasts.
\end{abstract}

Mots clés : video système terrestre, région d'influence d'un panache, panache pulsé 


\section{Introduction}

Les matières en suspension (MES), rejetées dans l'océan par un fleuve, sont souvent visualisées par la présence d'un panache turbide de surface. Ces MES peuvent être associées à des contaminants d'origine organique ou métallique. Il est donc nécessaire de comprendre les mécanismes qui contrôlent leur dispersion afin d'évaluer la qualité des eaux dans les régions adjacentes aux embouchures de fleuve.

Ces dernières décennies, un intérêt considérable a été porté sur le développement de techniques de télédétection destinées à estimer les dispersion des MES à partir des caractéristiques optiques des eaux de surfaces ([11], [1], [6], [8]). Les mesures multi-spectrales, par capteur satellite ou caméra aéroportée, permettent désormais de détecter la région influencée par un panache (RIP) turbide sur plusieurs dizaines de kilomètres. Cependant ces systèmes possèdent plusieurs limitations. La télédétection spatiale permet, au mieux, d'accéder à des données quotidiennes dont la qualité est fortement dépendante des caractéristiques orbitales du satellite et des conditions météorologiques. Bien que la télédétection aéroportée permette à l'utilisateur de définir les paramètres de déploiement de l'instrument (i.e. plan de vol, resolution spatio-temporelle), cette technique nécessite un matériel lourd et à un coût élevé. Récemment, les techniques vidéo ont été utilisées pour étudier la dynamique côtière [7]. Ces techniques permettent d'acquérir automatiquement des données à haute fréquence avec une résolution allant du centimètre à la centaine de mètres.

Cette étude présente une utilisation unique du système vidéo terrestre Argus (http://www.wldelft.nl/argus) pour suivre la dynamique spatiale d'un panache fluvial. Ce système a été installé à proximité de l'embouchure de l'Adour en février 2005. Les caractéristiques hydro-météorologiques du site d'étude sont exposées en première partie. Les différents forçages, susceptibles d'agir sur la dynamique du panache de l'Adour, sont présentés dans la section 3. Le contour du panache, obtenu à partir de l'analyse des images vidéo, pour différents événements météo-marins, est analysé en fonction des forçages dans la section 4. La dernière partie est consacrée aux conclusions et aux perspectives.

\section{Présentation du site d'étude}

\subsection{L'estuaire de l'Adour}

L'estuaire de l'Adour (Figure 1) a été choisi pour cette étude puisqu'il est représentatif des estuaires urbains modifiés par l'homme. Il a été canalisé de façon drastique au cours du dernier siècle. Il présente un canal étroit variant de $500 \mathrm{~m}$ en amont, à seulement $250 \mathrm{~m}$ au niveau de l'embouchure. Dans le bassin versant, le cours d'eau traverse des zones agricoles, puis des zones industrielles et urbaines à proximité de la ville de Bayonne. Le régime de marée est de type mésotidal semidiurne, de période $12 \mathrm{~h} 25$. L'amplitude moyenne de la marée est de 3,85 m en 
vives-eaux, et de $1,85 \mathrm{~m}$ en mortes eaux. Le littoral Angloy est soumis à des conditions de houles très énergétiques (régime estival : $\mathrm{Hs}=0,8$; régime hivernal : $\mathrm{Hs}=4,5 \mathrm{~m}$ ). L'effet de la houle sur le comportement du panache de l'Adour n'est pas pris en compte dans cette étude préliminaire.

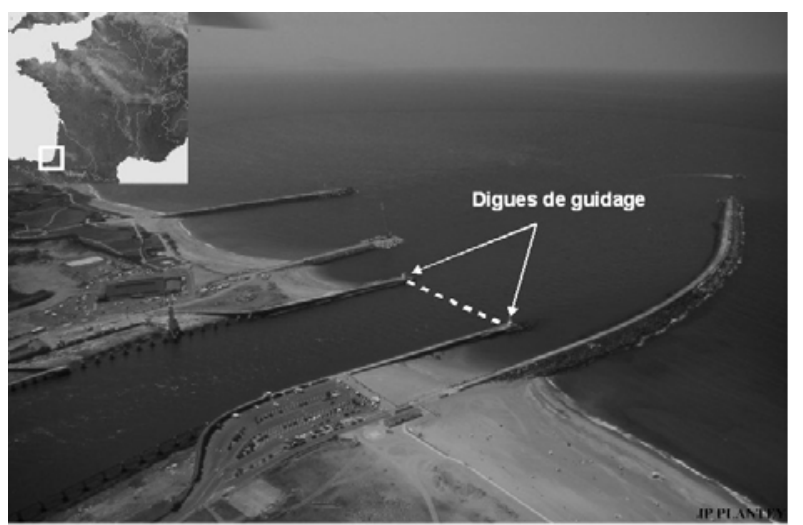

Figure 1- Situation géographique et aménagements de l'embouchure de l'Adour.

Les débits de l'Adour ont été calculés à partir des données de la DIREN sur une période de 75 ans (de 1930 à 2005). Le débit moyen annuel est de $300 \mathrm{~m}^{3} \cdot \mathrm{s}^{-1}$. Il peut atteindre $2000 \mathrm{~m}^{3} \cdot \mathrm{s}^{-1}$ pendant les périodes de crue. Les débits moyens mensuels sont présentés sur la figure 2. La période d'étiage s'étend du mois de juillet au mois de septembre, avec un débit moyen de $160 \mathrm{~m}^{3} \cdot \mathrm{s}^{-1}$. Pour cette période, la variation des débits moyens est faible $\left(\sigma_{\text {moy }} \sim+/-50 \mathrm{~m}^{3} . \mathrm{s}^{-1}\right)$. Les périodes de crue sont principalement présentes au cours de l'automne et de l'hiver. Toutefois, quelques évènements brusques peuvent apparaître au cours du printemps. Pour ces trois saisons, la variation des débits moyens est plus importante $\left(\sigma_{\text {moy }} \sim+/-200 \mathrm{~m}^{3} \cdot \mathrm{s}^{-1}\right)$.

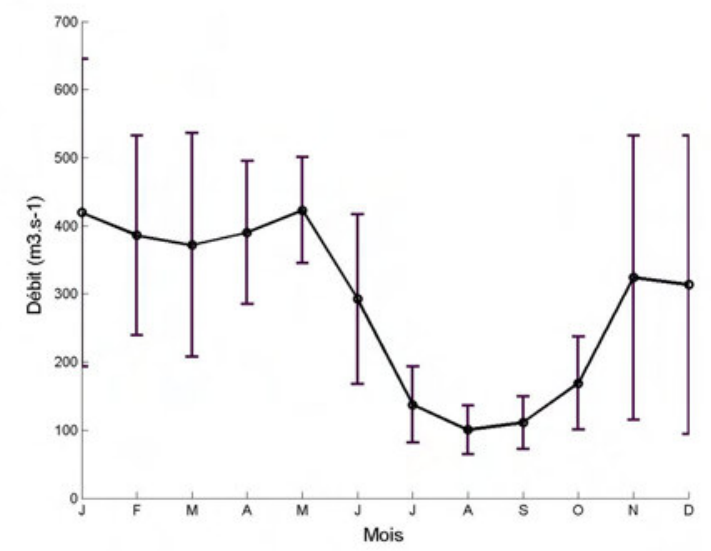

Figure 2- Débits moyens mensuels de l'Adour et leur écart type $(\sigma)$ de 1930 à 2005.

\subsection{Régime de vent}


Une analyse fréquentielle des vitesses et des directions du vent, mesurées à la station Météo France de Biarritz, a été réalisée sur un jeu de données couvrant une période de 15 ans (janvier 1991 à février 2006). Pour cette étude, le régime de vent au niveau du littoral d'Anglet est supposé identique à celui mesuré à Biarritz. Les résultats de l'analyse des données de vent sont représentés sur la figure 3 pour chaque saison. La vitesse moyenne est de $3,7 \mathrm{~m} . \mathrm{s}^{-1}$. Au cours de l'automne et de l'hiver, le littoral d'Anglet est majoritairement soumis à des vents de secteur sud, à dominante sud-ouest pour des vitesses supérieures à la moyenne. Le printemps et l'été, le régime de vent est majoritairement de secteur ouest, à nord-ouest. Pour cette période, la fréquence des vents dont la vitesse est supérieure à la moyenne, est deux fois plus importante.
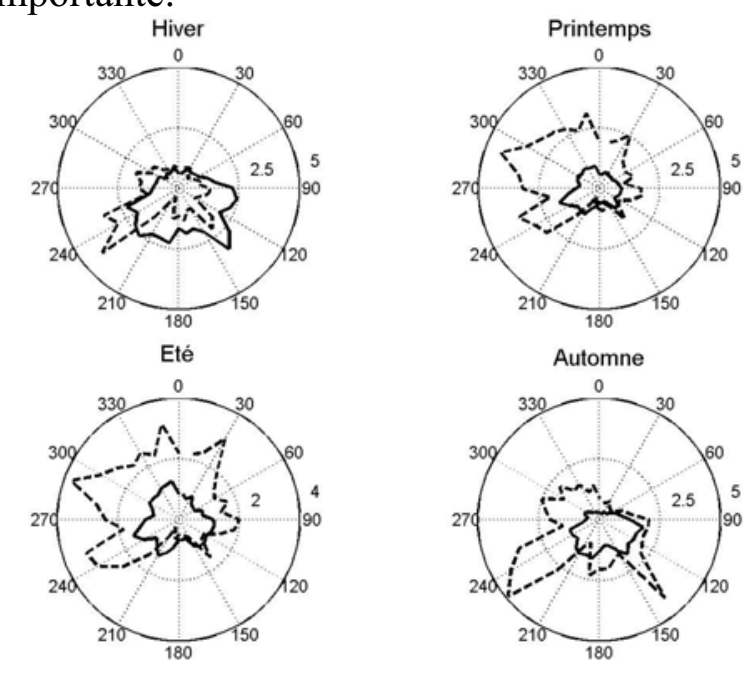

Figure 3- Pourcentages saisonniers des vents inférieurs à 3,7 $\mathrm{m} \cdot \mathrm{s}^{-1}$ (trait continu), et supérieurs à $3.7 \mathrm{~m} \cdot \mathrm{s}^{-1}$ (trait pointillé), en hiver (a), au printemps (b), en été (c), et à l'automne (d) (données moyennées sur une heure, fournies par Météo France).

\subsection{Courants de marée}

Des campagnes de mesures portants sur l'hydrodynamique côtière du littorale d'Anglet ([4], [2]), ont permis de montrer qu'au niveau de l'embouchure de l'Adour, pour des débits de 300 à $600 \mathrm{~m}^{3} . \mathrm{s}^{-1}$, et pour des coefficients de marée de 50 à 100 :

- les courants de jusant, orientés dans le sens de l'écoulement du fleuve, atteignent des vitesses supérieures à $1,5 \mathrm{~m} . \mathrm{s}^{-1}$,

- la renverse des courants de flot s'effectue 1 h30 après l'étale de marée basse. La stratification est bien marquée. L'Adour s'écoule sur la couche supérieure d'épaisseur $5 \mathrm{~m}$,

- les courants de flot atteignent des vitesses de $1,5 \mathrm{~m} \cdot \mathrm{s}^{-1}$,

- la renverse des courants à la fin du flot s'effectue $1 \mathrm{~h}$ après la pleine mer.

De plus, ces études ont permis de montrer que les courants de marée, en 
zone côtière, sont environ dix fois inférieur $\left(0,1 \mathrm{~m} \cdot \mathrm{s}^{-1}\right)$ à une distance de $500 \mathrm{~m}$ de l'embouchure.

\subsection{Densité de la colonne d'eau}

De façon générale, dans les régions soumises à une marée mésotidal, les flux entrants et sortants de marée entretiennent une dilution induite par un niveau de turbulence intense, qui tend à homogénéiser la colonne d'eau. Cependant, une campagne de mesures, réalisée par Cereca (Cellule d'Etude et de Recherche en Environnement estuarien et Côtier en Aquitaine) [3] en mai 1998, au niveau de l'embouchure de l'Adour, a permis de mettre en évidence un niveau élevé de stratification de la colonne d'eau. Des mesures de salinité et de température ont été réalisées au moyen d'une sonde $\mathrm{CTD}$, pendant les premières heures du flot, pour des débits de 300 à $600 \mathrm{~m}^{3} \cdot \mathrm{s}^{-1}$, et pour des coefficients de marée de 50 à 100 . Pour chacun des profils de salinité obtenus, on observe une forte stratification de la colonne d'eau caractérisée par la présence d'une halocline marquée. Sa profondeur varie en fonction du cycle de marée. Toutefois, elle est constante pour différents débits, à des niveaux de marée identiques. De plus, les eaux océaniques et fluviales ont respectivement une salinité moyenne de $33 \%$ et $1 \%$.

\section{Forces agissant sur la dynamique d'un panache fluvial}

Dans cette section, les forces agissant sur la dynamique d'un panache fluvial au niveau de l'embouchure sont différenciées des forces agissant sur la dynamique au niveau du plateau continental (dynamique côtière).

\subsection{Dynamique d'embouchure}

Dans les régions soumises à un fort régime de marée, la décharge du fleuve n'est pas constante. L'onde de marée se propage dans l'embouchure et perturbe l'écoulement du fleuve. Si le débit est faible, ou que l'amplitude de marée est importante, le transport peut être périodiquement inversé et dirigé vers l'amont du fleuve. Dans ce cas, les eaux fluviales rejetées sur le plateau continental pendant le précédent cycle de marée, se décrochent de l'embouchure. Le panache prend alors l'allure d'une forme "patatoïdale". La succession de ces évènements aboutit à un régime pulsatile du débit du fleuve, dont la fréquence correspond au cycle de marée. Cette caractéristique a été observée dans la région de l'embouchure de l'Adour à partir des images satellites MODIS, et des images vidéo Argus [9].

Selon Ruijter et al. [5], il existe deux critères qui déterminent la formation d'un débit pulsé :

- un critère de décrochement. Si la vitesse moyenne du débit du fleuve 
$\left(V_{D}\right)$ est inférieure à la vitesse des courants de marée $\left(V_{T}\right)$, l'onde de marée bloque le débit du fleuve périodiquement. Les conditions propres à la formation du panache ne sont alors plus réunies ;

- un critère de séparation. Si le rayon d'inertie $\left(R_{I}=V_{D} / f\right.$, où $f$ est le paramètre de Coriolis) est supérieur à la largeur de l'embouchure $\left(X_{R}\right)$, après un cycle tidal, la pulsation se sépare de l'embouchure suffisamment longtemps pour ne pas interférer avec la pulsation suivante.

La vitesse moyenne du débit d'un fleuve est estimée par :

$$
V_{D}=\frac{Q}{\left(H X_{R}\right)} \frac{S_{A}}{\left(S_{A}-S_{D}\right)} \frac{1}{(1-\Delta)}
$$

où $Q$ est le débit du fleuve, $H$ l'épaisseur de la couche de surface, $X_{R}$ la largeur de l'estuaire, $S_{A}\left(S_{D}\right)$ la salinité des eaux océaniques (fluviales), et où $\Delta$ est la fraction de la période de marée pendant laquelle le flux est inversé, et est approximé par :

$$
\Delta=\frac{1}{\pi} \arccos \left(\frac{V_{D}}{V_{T}}\right)=\frac{1}{\pi} \arccos \left(\frac{Q}{\left(H X_{R} V_{T}\right)} \frac{S_{A}}{\left(S_{A}-S_{D}\right)}\right)
$$

où $V_{T}$ est la vitesse du courant de marée au niveau de l'embouchure.

Les critères de pulsation du panache de l'Adour ont été calculés (Figure 4) à partir des équations (1) et (2), en considérant un transect $X_{R}=250 \mathrm{~m}$, situé entre l'extrémité des deux digues de guidage (Figure 1), une vitesse de courant de marée $V_{T}=1,5 \mathrm{~m} . \mathrm{s}^{-1}$ (section 2.3), une couche supérieure d'épaisseur moyenne $H=5 \mathrm{~m}$ (section 2.4), et pour des débits $Q$ de 300 à $2000 \mathrm{~m}^{3} \cdot \mathrm{s}^{-1}$. Les critères de décrochement et de séparation indiquent que l'Adour possède des caractéristiques pulsatiles pour des débits compris entre 300 et $900 \mathrm{~m}^{3} \cdot \mathrm{s}^{-1}$. Pour des débits supérieurs à $900 \mathrm{~m}^{3} . \mathrm{s}^{-1}$, la vitesse moyenne du débit du fleuve est trop importante par rapport à la vitesse du courant de marée, et le débit de l'Adour ne sera pas pulsé $\left(V_{D} / V_{T}>1\right)$.

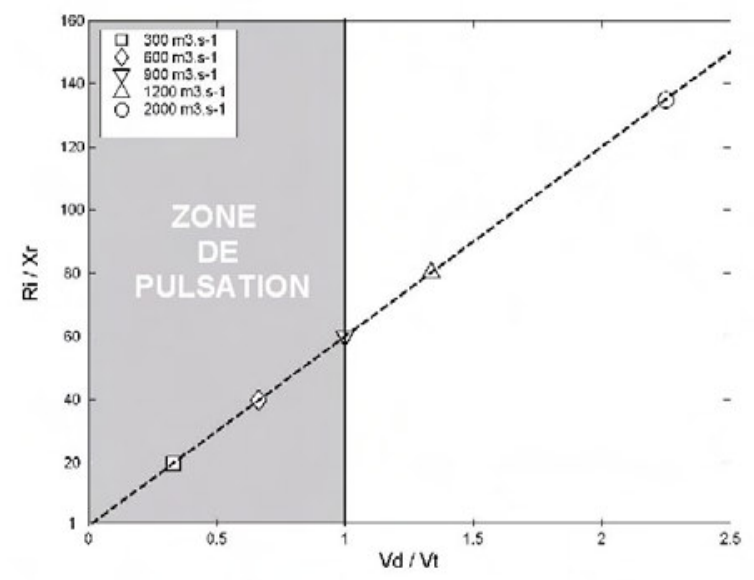

Figure 4- Représentation des critères de décrochement (abscisse) et de séparation (ordonnée) pour l'estuaire de l'Adour.

\subsection{Dynamique côtière}


En supposant un mouvement quasi-horizontal, les principales forces agissant sur une particule d'eau au sein d'un panache fluvial sont : la force centrifuge $\vec{f}_{r}$, la force de Coriolis $\vec{f}_{c}$, et la force du vent $\vec{f}_{w}$. Deux nombres adimensionnels permettent de décrire l'amplitude relative des composantes transverses de ces forces :

- le nombre de Rossby $N_{R}$, qui correspond au rapport de la force centrifuge sur la force de Coriolis :

$$
N_{R}=\frac{f_{r}}{f_{c}}=\left(\frac{U^{2}}{R}\right)\left(\frac{1}{f U}\right)=\frac{U}{R f},
$$

où $U$ est la vitesse de l'écoulement, $R$ le rayon de courbure et $f$ le paramètre de Coriolis (proche de $1.10^{-4} \mathrm{~s}^{-1}$ ),

- le rapport de la force du vent sur la force centrifuge est exprimé par :

$$
Q_{W}=\frac{f_{w}}{f_{r}}=\frac{\rho_{a}}{\rho} C_{d}\left(\frac{w}{U}\right)^{2} \frac{R}{H}=K_{w}\left(\frac{w}{U}\right) \frac{R}{H},
$$

où $K_{w}$ est de l'ordre de $1.7 .10^{-6}$, et $w$ est la vitesse du vent.

Les valeurs des rapports $Q_{R}=1 / N_{R}$ et $Q_{W}$, calculées pour différents évènements de propagation du panache de l'Adour, sont comparées dans les sections 4.2 et 4.3 pour quantifier l'impact de chacune des forces.

\section{Application du système vidéo à la détection du panache de l'Adour}

Des images enregistrées par la station Argus d'Anglet, au cours de l'année 2005, ont été traitées à partir d'un algorithme de segmentation de pixels [10] dans le but de délimiter le contour du panache de l'Adour. Trois cas de propagation, détectées par cette méthode, sont analysés afin d'évaluer l'influence des trois principales forces qui contrôlent sa dynamique : la marée, la force de Coriolis, et le vent. Les résultats des détections de contour sont projetés sur une orthophotographie (fournie par l'IGN) de la région côtière d'Anglet, et de la zone d'embouchure de l'Adour (Figures 5, 6 et 7).

\subsection{Influence de la marée}

Le 19 mai 2005, pour un coefficient de marée de 51, une pleine mer atteinte à $12 \mathrm{~h} 35 \mathrm{GMT}$, et un débit de $360 \mathrm{~m}^{3} \cdot \mathrm{s}^{-1}$, un panache est détectable sur les images de la station Argus de $9 \mathrm{~h}$ à $14 \mathrm{~h}$ GMT. Les résultats sont présentés sur la figure 5 .

Pour un débit d'environ $300 \mathrm{~m}^{3} \cdot \mathrm{s}^{-1}$, les critères de pulsation sont vérifiés (Figure 4). La pulsation du panache est parfaitement identifiable. La propagation sud-ouest initialement établie au cours du jusant (Figure 5a), est perturbée une heure avant le niveau de pleine mer (Figure 5b). A 11h GMT, le système vidéo enregistre la formation d'un front au niveau de l'embouchure alors qu'il détecte 
encore la présence d'une nappe turbide d'orientation ouest nord-ouest (trait pointillé). A l'étale de marée haute (Figure 5c), un seul front est détecté au niveau de l'embouchure. L'onde de marée, qui se propage dans l'estuaire, bloque le débit de l'Adour pendant un laps de temps d'environ une heure à l'étale de marée haute. Puis, un nouveau lobe se forme pendant le début du flot (Figure 5d) et tend à se propager vers l'ouest.

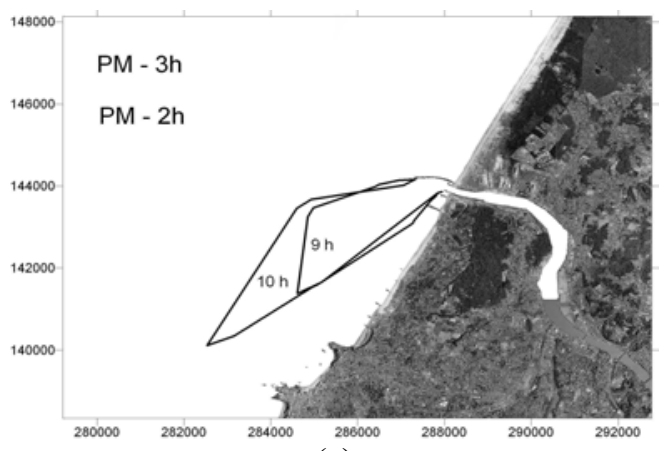

(a)

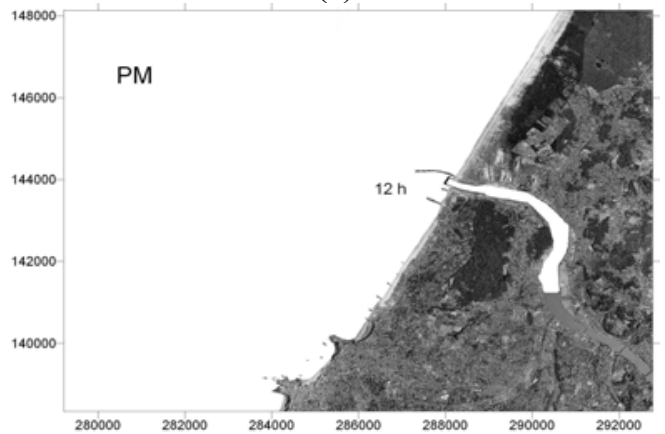

(c)

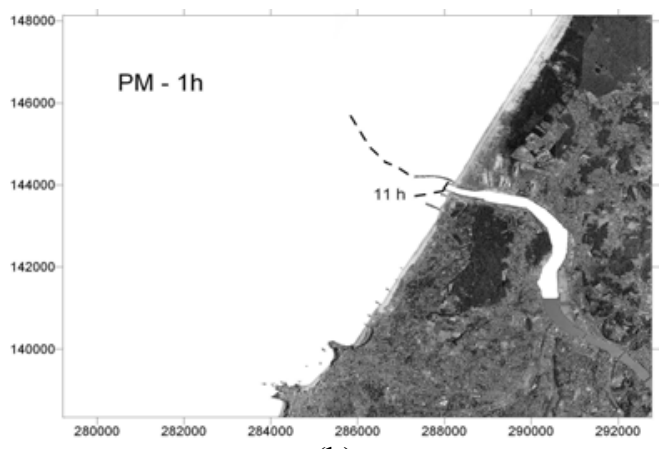

(b)

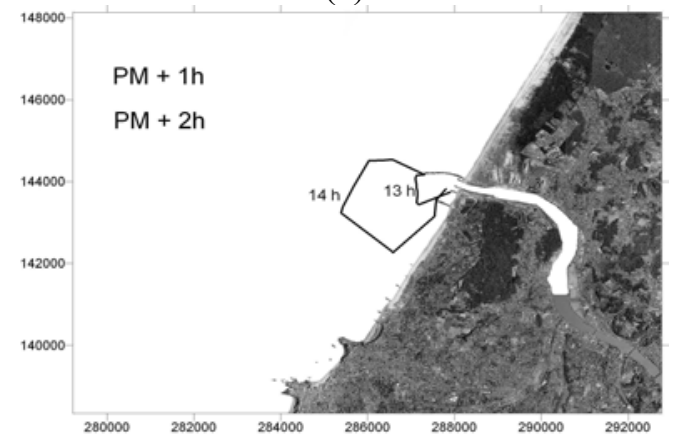

(d)

Figure 5- Propagation de surface du panache de l'Adour au jusant (a), à la fin du flot (b), à l'étale de marée haute (c), et au début du flot (d), pour un débit de $360 \mathrm{~m}^{3} . \mathrm{s}^{-1}$ et des vents de secteur sud est.

\subsection{Influence de la force de Coriolis}

Le 26 avril 2005, pour un coefficient de marée de 95, une pleine mer atteinte à $4 \mathrm{~h} 34 \mathrm{GMT}$, et un débit de $600 \mathrm{~m}^{3} . \mathrm{s}^{-1}$, un panache est observable sur les images de la station Argus de $6 \mathrm{~h}$ à $8 \mathrm{~h}$ GMT. Les résultats sont présentés sur la figure 6 .

A 6h GMT, le panache est situé à proximité de l'embouchure. Il s'étend longitudinalement sur $1 \mathrm{~km}$ et transversalement sur $2,8 \mathrm{~km}$. Deux heures plus tard, la dispersion transverse et longitudinale s'est faite sur $3 \mathrm{~km}$. A partir de ces résultats, la vitesse $U$ de propagation du panache a été estimée. Les rapports $Q_{R}$ et $Q_{W}$ (section 3.2) ont été calculés (Tableau 1) en considérant une valeur approximative $H$ de la profondeur du panache. Cette valeur a été estimée à partir de la campagne Cereca [8] en raison du manque de données in-situ. A mi-marée, les données indiques que la profondeur du panache est d'environ $2 \mathrm{~m}$. Pour des 
vents de 2 à $4 \mathrm{~m} \cdot \mathrm{s}^{-1}, Q_{R}$ est environ 10 fois supérieure à $Q_{W}$. Dans ce cas, la force de Coriolis contrôle la dynamique du panache et dévie les masses d'eau en mouvement vers la droite (hémisphère nord). La forme "patatoïdale" du panache a tendance à se propager vers l'ouest et le nord.

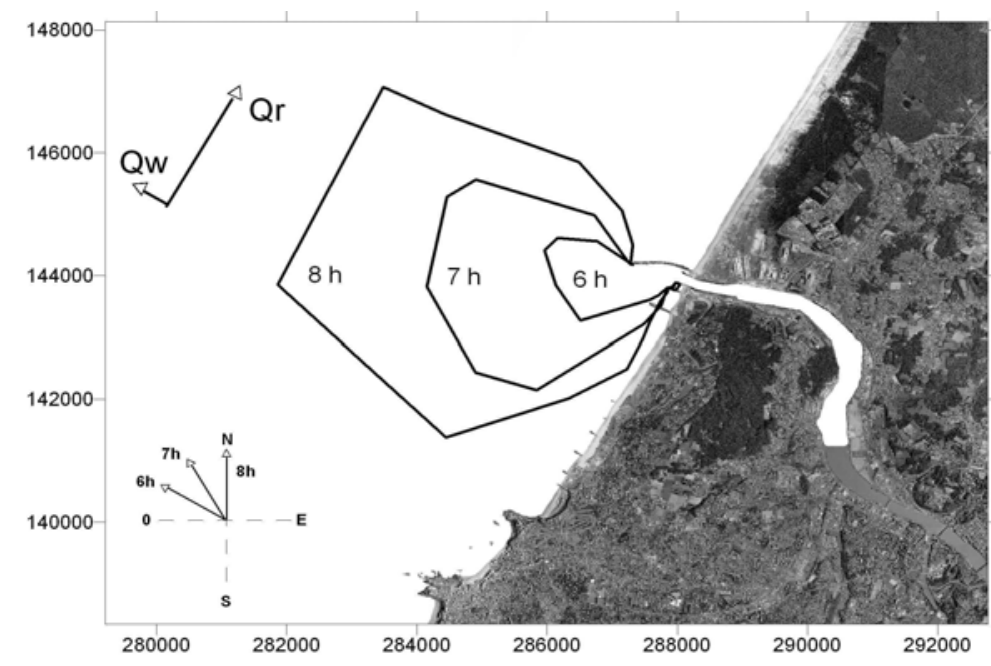

Figure 6- Propagation du panache de l'Adour, au cours du jusant, le 26 avril 2005 de 6h à 8h GMT, pour un débit de $300 \mathrm{~m}^{3} . \mathrm{s}^{-1}$. La direction du vent pour chacune des heures est indiquée par une flèche en bas à gauche de la figure.

\begin{tabular}{|c|c|c|c|c|c|c|}
\hline Date & Heures & $\boldsymbol{W}\left(\boldsymbol{m} \cdot \boldsymbol{s}^{-1}\right)$ & $\boldsymbol{U}\left(\boldsymbol{m} \cdot \boldsymbol{s}^{-1}\right)$ & $\boldsymbol{R}(\boldsymbol{m})$ & $\boldsymbol{Q}_{\boldsymbol{R}}$ & $\boldsymbol{Q}_{W}$ \\
\hline \multirow{2}{*}{$26 / 04 / 05$} & $6-7 \mathrm{~h}$ & 2 & 0,6 & 390 & $\mathbf{0 , 0 7}$ & 0,003 \\
\cline { 2 - 7 } & $7-8 \mathrm{~h}$ & 4 & 0,7 & 820 & $\mathbf{0 , 1}$ & 0,02 \\
\hline \multirow{3}{*}{$15 / 05 / 05$} & $8-9 \mathrm{~h}$ & 12 & 0,2 & 515 & 0,3 & $\mathbf{1 , 8}$ \\
\cline { 2 - 7 } & $9-10 \mathrm{~h}$ & 12 & 0,4 & 755 & 0,2 & $\mathbf{0 , 5}$ \\
\cline { 2 - 7 } & $10-11 \mathrm{~h}$ & 14 & 0,6 & 1420 & 0,2 & $\mathbf{0 , 7}$ \\
\hline
\end{tabular}

Tableau 1- Echelle des différentes forces agissant sur le panache de l'Adour au cours des deux cas d'étude : le 26 avril 2005 et le 15 mai 2005.

\subsection{Influence du vent}

Le 15 mai 2005, pour un coefficient de marée de 39, une pleine mer atteinte à $8 \mathrm{~h} 14 \mathrm{GMT}$, et un débit de $300 \mathrm{~m}^{3} \cdot \mathrm{s}^{-1}$, un panache est observable sur les images de la station Argus de $8 \mathrm{~h}$ à $11 \mathrm{~h}$ GMT. Les résultats sont présentés sur la figure 7.

A 8h GMT, l'action des courants de marée, en fin de flot, maintient les eaux turbides dans la zone de l'embouchure. De $9 \mathrm{~h}$ à $11 \mathrm{~h}$ GMT, le panache se propage transversalement sur $2 \mathrm{~km}$ et longitudinalement sur $4 \mathrm{~km}$. La comparaison des valeurs de $Q_{R}$ et $Q_{W}$, calculées pour chaque propagation avec $H=$ $2 \mathrm{~m}$ (Section 4.2), montre que la force du vent est supérieure à la force de Coriolis 
dans chacun des cas (Tableau 1). La forme "patatoïdale", beaucoup moins marqué sur cet évènement, se propage en longeant les côtes d'Anglet, vers le sud-ouest sous l'action des vents de secteur nord à nord-est.

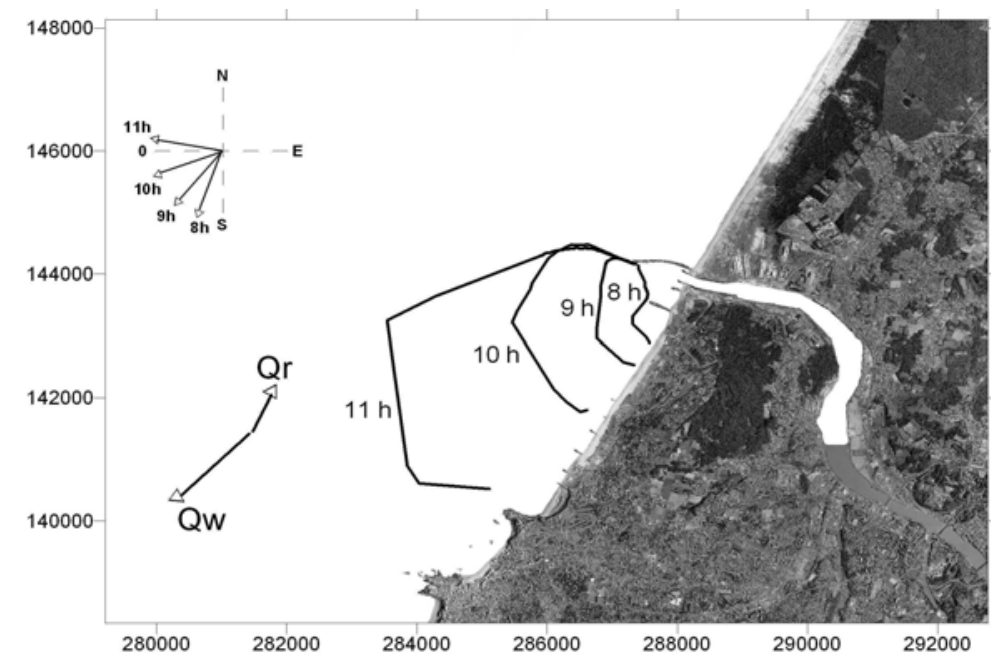

Figure 7- Propagation du panache de l'Adour, au cours du jusant, le 19 avril 2005 de $8 \mathrm{~h}$ à $11 \mathrm{~h}$ GMT, pour un débit de $300 \mathrm{~m}^{3} \cdot \mathrm{s}^{-1}$. La direction du vent pour chacune des heures est indiquée par une flèche en haut à gauche de la figure.

\section{Conclusions et perspectives}

Les résultats préliminaires mettent en évidence le caractère pulsatile du panache de l'Adour. L'estimation de critères de pulsations, basés sur les caractéristiques hydro-morphologiques de l'embouchure de l'Adour, montrent que ce comportement est susceptible de se produire pour des débits inférieurs à 900 $\mathrm{m}^{3} \cdot \mathrm{s}^{-1}$. Une étude comparative de l'influence de la force de Coriolis et du vent a été réalisée. Pour une faible intensité de vent, le panache va former un bombement anticyclonique qui se propage vers le nord-ouest, sous l'influence de la force de Coriolis. Au contraire, pour une forte intensité de vent, la force de Coriolis est moins influente. Dans ce cas, la propagation du panache a tendance à longer les côtes pour des vents de secteur nord (automne, hiver), et à s'en éloigner pour des vents de secteur sud (printemps, été).

Il est nécessaire de réaliser des campagnes de mesures complémentaires afin d'évaluer plus précisément la stratification de la colonne d'eau et l'hydrodynamique sur toute la surface du panache. Ces informations permettront d'améliorer la description de son comportement en fonction des conditions hydrométéorologiques locales.

Cette étude a également montré que les données horaires, fournies par le système video terrestre, permettent de réaliser une analyse précise de l'impact des différents forçages qui contrôlent la dynamique côtière d'un panache fluvial pour des régimes de marée mésotidal. Ce système, couplé à une analyse par 
télédétection spatiale (MODIS), devrait permettre de définir la RIP de l'Adour à plus grande échelle.

\section{Bibliographie}

[1] Baban S. M. J., (1995). The use of Landsat imagery to map fluvial sediment discharge into coastal waters. Marine Geology, 123, 263-270.

[2] Brière C., (2005). Etude de l'hydrodynamique d'une zone côtière anthropisée : l'embouchure de l'Adour et les plages adjacentes d'Anglet. Thèse d'hydraulique maritime et transport sédimentaire, LaSAGeC2, Université de Pau et des Pays de l'Adour, 99-141.

[3] Cereca, Cellule de valorisation de l'Ifremer, (1998). Campagne de mesures: salinité et température dans l'estuaire de l'Adour.

[4] CreOcean, (1997). Etude d'impact des travaux de rectification de l'embouchure de l'Adour. Rapport interne.

[5] De Ruijter W. P. M., Visser A.W., et Bos W.G., (1997). The Rhine outflow : A prototypical pulsed discharge plume in a high energy shallow sea. Journal of Marine System, 12, 263-276.

[6] Froidefond J. M., Lahet F., Hu C., Doxaran D., Guiral D., Prost M.T., et Ternon M.T., (2004). Mudflats and mud suspension observed from satellite data in French Guiana. Marine Geology, 208, 153-168.

[7] Holman, R.A. et Stanley, J. (in press). The history, capabilities and recent developments of Argus. Accepted for publication in Coastal Engineering.

[8] Miller R. L. et McKee B. A., (2004). Using MODIS Terra $250 \mathrm{~m}$ imagery to map concentrations of total suspended matter in coastal waters. Remote Sensing of Environment, 93, 259-266.

[9] Morichon D. et Dailloux D., (2006). River plume monitoring using two combined remote sensing techniques: satellite (MODIS) and video (ARGUS) images. Application to the Adour river plume (France). Accepted for Proceeding in ICCE San Diego.

[10] Morichon D., Dailloux D., Aarninkhof S. Using a shore-based video system (ARGUS) to monitor turbid river plume front. Submitted to Journal of Coastal Research.

[11] Tassan S., (1994). Local algorithms using SeaWiFS data for retrieval of phytoplankton, pigments, suspended sediment, and yellow susbstence in the coastal water. Applied Optics, 33, 2369-2378. 